\title{
Classification of homotopy classes of equivariant gradient maps
}

\author{
by \\ E. N. Dancer (Sydney), K. Gęba (Gdańsk) and \\ S. M. Rybicki (Toruń)
}

\begin{abstract}
Let $V$ be an orthogonal representation of a compact Lie group $G$ and let $S(V), D(V)$ be the unit sphere and disc of $V$, respectively. If $F: V \rightarrow \mathbb{R}$ is a $G$-invariant $C^{1}$-map then the $G$-equivariant gradient $C^{0}$-map $\nabla F: V \rightarrow V$ is said to be admissible provided that $(\nabla F)^{-1}(0) \cap S(V)=\emptyset$. We classify the homotopy classes of admissible $G$-equivariant gradient maps $\nabla F:(D(V), S(V)) \rightarrow(V, V \backslash\{0\})$.
\end{abstract}

1. Introduction. The purpose of this paper is to understand the topological invariants associated with equivariant gradient maps.

More precisely, we assume $V$ is a real finite-dimensional orthogonal representation of a compact Lie group $G$. In other words, $V$ is a real finitedimensional linear space with a scalar product and there is an orthogonal action of $G$ on $V$ denoted by $g x$ for $g \in G, x \in V$. We are then interested in the homotopy classes of $G$-equivariant gradient mappings from $V$ into $V$, which are non-zero on the unit sphere $S(V)$ of $V$. Here a map $\nabla F: V \rightarrow V$ is said to be $G$-equivariant if $\nabla F(g x)=g \nabla F(x)$ for $g \in G, x \in V$. We completely classify the homotopy classes of such maps in terms of a $G$-equivariant gradient degree defined here. Our gradient degree is a natural generalization of the classical Brouwer degree with the ring of integers replaced by a ring $U(G)$ determined by the orbit types of $G$ (see [4] for the definition of $U(G)$ ).

Results of this type are of considerable interest because they show that there are no extra invariants to be found. Note that the study of the homotopy classes of $G$-equivariant gradient maps is a natural problem as these maps arise in many applications (see $[3,10,11,12]$ for example). The stronger homotopy invariance properties of our degree are reflected in the possibility

2000 Mathematics Subject Classification: 47H11, 55P91.

Key words and phrases: equivariant degree, classification of gradient equivariant homotopy classes.

Research of S. M. Rybicki supported by the State Committee for Scientific Research, Poland, under grant No. 5 PO3A 02620. 
to obtain connected sets of bifurcation solutions as in $[3,10,11,12]$. The Conley index is not suitable for this.

The analogous problem of homotopy classification of $G$-equivariant mappings when there is no gradient structure is well known in $G$-equivariant topology. In this case classifying our homotopies is equivalent to classifying the equivariant maps of $S(V)$ into itself. It turns out that in this case the classical Brouwer degree, which assigns an integer to each homotopy class, can be replaced by a more refined invariant which assigns to a homotopy class an element of the Burnside ring $A(G)$ of $G$. In most (but not all) cases, this new invariant is determined by the Brouwer degrees of the induced maps on fixed point subspaces of isotropy subgroups (see [4, 9] and the references therein).

In the case $G=\{e\}, V=\mathbb{R}^{n}$ Parusiński proved that the classical Brouwer degree is the only invariant (see [8]). More precisely, he showed that two gradient maps non-vanishing on the unit sphere $S^{n-1}$ are homotopic as gradient maps if and only if they are homotopic in the ordinary sense.

On the other hand, the first named author essentially showed in [3] that for the group $S O(2)$, the analogue of the Parusiński result for $S O(2)$ equivariant gradient maps does not hold and he constructed an extra invariant. The work was further improved in [10] for $S O(2)$-actions and in [6] for general compact Lie group actions.

In the proof of the main result of [8] the following construction plays a fundamental role: assume that $F=\nabla \varphi$ is a gradient $C^{0}$-map on $\mathbb{R}^{n}$. For $x \in S^{n-1}$ we have $F(x)=F_{0}(x)+f(x) x$, where $F_{0}=\nabla\left(\varphi_{\mid S^{n-1}}\right)$. Then $F$ is non-vanishing on $S^{n-1}$ if and only if $F_{0}^{-1}(0) \cap f^{-1}(0)=\emptyset$. Thus to an admissible gradient vector field $F$ on $\mathbb{R}^{n}$ there corresponds the gradient pair $\left(F_{0}, f\right)$ which "lives" on the $(n-1)$-dimensional sphere. In this way Parusiński reduces dimension.

In our paper, generalizing the above construction, we introduce the notion of equivariant gradient pair. More precisely, given a smooth Riemannian manifold $M$ with a smooth action of a compact Lie group $G$ we consider pairs $(X, f)$, where $X$ is a $G$-equivariant gradient vector field on $M$ and $f$ is a $G$-invariant function on $M$. There is also a natural notion of homotopy between $G$-equivariant gradient pairs.

In this article we consider smooth Riemannian $G$-manifolds $M$ with a finite number of orbit types, i.e. $\# \Phi(M)<\infty$ (see Section 2), and notice that if $\Phi(M)=\{(H)\}$ then there is a bijection between the homotopy classes of $G$-equivariant gradient pairs on $M=M_{(H)}$ and the homotopy classes of gradient pairs on the quotient manifold $M_{(H)} / G$. Next combining Corollary 3.1 and Corollary 6.2 we classify the homotopy classes of equivariant gradient pairs on $M$. 
In Section 2 we recall some definitions of equivariant topology and give the precise definitions used in our paper. Section 3 contains the basic technical results. The main results of this section are Theorem 3.1 and Corollary 3.1. Section 4 contains the main results of this paper. Theorem 4.1 gives the classification of homotopy classes of $G$-equivariant gradient pairs on $M$ and Corollary 4.1 gives the classification of homotopy classes of admissible (non-vanishing on $S(V)$ ) G-equivariant gradient maps. In Section 5 we hint at some relation between the $G$-equivariant Conley index theory and the $G$-equivariant gradient degree. In the Appendix we classify the homotopy classes of gradient pairs on $N$, where $N$ is a connected smooth Riemannian manifold with $\operatorname{dim} N \geq 1$. We discuss Parusiński's proof of the above mentioned result (see [8]). Essentially we rewrite this proof using the language of gradient pairs. We are convinced that understanding this approach may result in a better understanding of the technicalities of our paper.

The authors thank the referees for their helpful suggestions concerning the presentation of this paper.

2. Equivariant gradient maps and pairs. In this section we set up notation and terminology. Throughout this article $G$ stands for a compact Lie group, and $V$ denotes a real, finite-dimensional, orthogonal representation of $G$ with $G$-invariant scalar product $\langle\cdot, \cdot\rangle$ and norm $|\cdot|$. Set $D(V)=\{v \in V$ : $|v| \leq 1\}, B(V)=\{v \in V:|v|<1\}$ and $S(V)=\{v \in V:|v|=1\}$. Since the representation $V$ is orthogonal, these sets are $G$-invariant.

Moreover, we will denote by $M$ a Riemannian smooth $G$-manifold. Since the group $G$ is compact, without loss of generality one can assume that $M$ is equipped with a $G$-invariant metric $\langle\cdot, \cdot\rangle$. The action of the group $G$ on $M \times[0,1]$ is given by $g(v, t)=(g v, t)$ for any $(g, v, t) \in G \times M \times[0,1]$. The tangent bundle $T(M)$ is considered as a $G$-bundle with the induced orthogonal $G$-action.

We will denote by $\nabla \varphi: M \rightarrow T(M)$ the gradient of a $C^{1}$-map $\varphi$ : $M \rightarrow \mathbb{R}$. The class of $G$-invariant $C^{k}$-maps $\varphi: M \rightarrow \mathbb{R}$ will be denoted by $C_{G}^{k}(M, \mathbb{R})$. It is known that if $\varphi \in C_{G}^{1}(M, \mathbb{R})$, then $\nabla \varphi: M \rightarrow T(M)$ is a $G$-equivariant continuous vector field.

The set of conjugacy classes of closed subgroups of $G$ will be denoted by $\Phi(G)$ and the conjugacy class of a closed subgroup $H \subset G$ will be denoted by $(H)$. A subgroup $H \subset G$ is subconjugate to a subgroup $K \subset G$ (written $(H)<(K))$ if $H$ is conjugate to a subgroup of $K$. Subconjugation defines a partial ordering on $\Phi(G)$.

Let $G_{v}=\{g \in G: g v=v\}$ be the isotropy subgroup of $v \in M$. For $(H) \in$ $\Phi(G)$ we set $M_{(H)}=\left\{v \in M:\left(G_{v}\right)=(H)\right\}$ and $\Phi(M)=\{(H) \in \Phi(G)$ : $\left.M_{(H)} \neq \emptyset\right\}$. Notice that $M_{(H)}$ is a submanifold of $M$ and the quotient map $\pi_{(H)}: M_{(H)} \rightarrow M_{(H)} / G$ is smooth. Moreover, the orbit $G \cdot v$ is a submanifold 
of $M_{\left(G_{v}\right)}$ diffeomorphic to $G / G_{v}$. We will denote by $\Psi_{(H)}(M)$ the family of connected components of $M_{(H)} / G$ and $\Psi(M)=\bigcup_{(H) \in \Phi(M)} \Psi_{(H)}(M)$.

Definition 2.1. Let $F \in C_{G}^{1}(V, \mathbb{R})$. A map $\nabla F \in C_{G}^{0}(V, V)$ is called a $G$-equivariant admissible gradient map (written $\nabla F \in \mathcal{G}(V)$ ) if $(\nabla F)^{-1}(0) \cap$ $S(V)=\emptyset$.

Definition 2.2. Let $H \in C_{G}^{1}(V \times[0,1], \mathbb{R})$. The map $\nabla_{v} H: V \times[0,1] \rightarrow$ $V$ is called a $\mathcal{G}(V)$-homotopy if $\left(\nabla_{v} H\right)^{-1}(0) \cap(S(V) \times[0,1])=\emptyset$. We say that maps $\nabla F_{0}, \nabla F_{1} \in \mathcal{G}(V)$ are $\mathcal{G}(V)$-homotopic if there is a $\mathcal{G}(V)$-homotopy $\nabla_{v} H: V \times[0,1] \rightarrow V$ such that $\nabla_{v} H(\cdot, i)=\nabla F_{i}(\cdot)$ for $i=0,1$.

Let $\nabla F \in \mathcal{G}(V)$. For $v \in S(V)$ we have the unique decomposition $\nabla F(v)=(\nabla F(v)-\langle\nabla F(v), v\rangle \cdot v)+\langle\nabla F(v), v\rangle \cdot v=X_{\nabla F}(v)+f_{\nabla F}(v) \cdot v$, where $X_{\nabla F}=\nabla\left(F_{\mid S(V)}\right)$ and $f_{\nabla F} \in C_{G}^{0}(S(V), \mathbb{R})$.

Similarly, if $\nabla H$ is a $\mathcal{G}(V)$-homotopy then for $v \in S(V)$ we have the decomposition

$$
\begin{aligned}
\nabla_{v} H(v, t) & =\left(\nabla_{v} H(v, t)-\left\langle\nabla_{v} H(v, t), v\right\rangle \cdot v\right)+\left\langle\nabla_{v} H(v, t), v\right\rangle \cdot v \\
& =X_{\nabla H}(v, t)+f_{\nabla H}(v, t) \cdot v
\end{aligned}
$$

where $X_{\nabla H}(\cdot, t)=\nabla\left(H(\cdot, t)_{\mid S(V)}\right)$ and $f_{\nabla H} \in C_{G}^{0}(S(V) \times[0,1], \mathbb{R})$.

The above leads us to the following definitions.

DeFinition 2.3. We say that $(X, f)$ is an equivariant gradient pair on $M$ if

- $f \in C_{G}^{0}(M, \mathbb{R})$,

- $N_{-}(f)=\{x \in M: f(x) \leq 0\}$ is a compact subset of $M$,

- $X: M \rightarrow T M$ and there is $\varphi \in C_{G}^{1}(M, \mathbb{R})$ such that $X=\nabla \varphi$,

- $X^{-1}(0) \cap f^{-1}(0)=\emptyset$.

The class of equivariant gradient pairs on $M$ will be denoted by $\mathcal{P}(M)$.

DeFINITION 2.4. We say that $(X, f)$ is an equivariant gradient pair homotopy on $M$ if

- $X: M \times[0,1] \rightarrow T M$ and there is $\varphi \in C_{G}^{1}(M \times[0,1], \mathbb{R})$ such that $X(\cdot, t)=\nabla_{x} \varphi(\cdot, t)$ for $t \in[0,1]$,

- $f \in C_{G}^{0}(M \times[0,1], \mathbb{R})$,

- $N_{-}(f)=\{x \in M \times[0,1]: f(x, t) \leq 0\}$ is a compact subset of $M \times[0,1]$, - $X^{-1}(0) \cap f^{-1}(0)=\emptyset$.

Equivariant gradient pair homotopies will be called $\mathcal{P}(M)$-homotopies.

From now on the set of $\mathcal{G}(V)$-homotopy classes will be denoted by $\mathcal{G}[V]$. Moreover, the set of $\mathcal{P}(M)$-homotopy classes in $\mathcal{P}(M)$ will be denoted by $\mathcal{P}[M]$. The principal significance of the next theorem is that it allows us to 
reduce the classification of $\mathcal{G}[V]$ to the classification of $\mathcal{P}[S(V)]$. In fact it is the first step in the classification of $\mathcal{G}[V]$.

LeMma 2.1. The map $\Upsilon_{V}: \mathcal{G}[V] \rightarrow \mathcal{P}[S(V)]$ defined by $\Upsilon_{V}([\nabla F])=$ $\left[\left(X_{\nabla F}, f_{\nabla F}\right)\right]$ is a bijection.

Lemma 2.1 is an equivariant version of Lemma 1 of [8] with the same proof, therefore we omit it.

Lemma 2.2. Let $M_{0}, M_{1}$ be Riemannian smooth $G$-manifolds with $G$-invariant metrics such that there exists a G-equivariant diffeomorphism $\Phi$ : $M_{0} \rightarrow M_{1}$. Then the map $\Phi^{*}: \mathcal{P}\left(M_{1}\right) \rightarrow \mathcal{P}\left(M_{0}\right)$ given by $\Phi^{*}(\nabla \varphi, f)=$ $(\nabla(\varphi \circ \Phi), f \circ \Phi)$ induces a bijection $\left[\Phi^{*}\right]: \mathcal{P}\left[M_{1}\right] \rightarrow \mathcal{P}\left[M_{0}\right]$.

The easy proof of this lemma is left to the reader.

Definition 2.5. We denote by $\mathcal{K}(M)$ the collection of pairs $(U, X)$ such that

- $U \subset M$ is open invariant;

- there is $\varphi \in C_{G}^{1}(U)$ such that $X=\nabla \varphi$;

- $X^{-1}(0)$ is a compact subset of $U$.

Definition 2.6. We say that $(U, X)$ is a $\mathcal{K}(M)$-homotopy if

- $U \subset M \times[0,1]$ is open invariant;

- there is $\varphi \in C_{G}^{1}(U)$ such that $X=\nabla_{x} \varphi$;

- $X^{-1}(0)$ is a compact subset of $U$.

Two pairs $\left(U_{0}, X_{0}\right),\left(U_{1}, X_{1}\right) \in \mathcal{K}(M)$ are said to be $\mathcal{K}(M)$-homotopic if there exists a $\mathcal{K}(M)$-homotopy $(U, X)$ such that, for $i=0,1$,

$$
U_{i}=\{x \in M:(x, i) \in U\}, \quad X_{i}(x)=X(x, i) .
$$

In this case $(U, X)$ is called a $\mathcal{K}(M)$-homotopy connecting $\left(U_{0}, X_{0}\right)$ and $\left(U_{1}, X_{1}\right)$. We denote by $\mathcal{K}[M]$ the set of all $\mathcal{K}(M)$-homotopy classes.

The following lemma will be useful in our considerations.

Lemma 2.3. Let $(U, X) \in \mathcal{K}(M)$. If $W \subset U$ is an open invariant subset and $X^{-1}(0) \subset W$ then

(a) $\left(W, X_{\mid W}\right) \in \mathcal{K}(M)$;

(b) $(U, X)$ and $\left(W, X_{\mid W}\right)$ are $\mathcal{K}(M)$-homotopic.

Proof. (a) Obvious.

(b) Set $V=U \times[0,1] \backslash(U \backslash W) \times\{1\}$ and $Y(x, t)=X(x)$ for $(x, t) \in V$. Evidently $(Y, V)$ is a $\mathcal{K}(M)$-homotopy connecting $(U, X)$ and $\left(W, X_{\mid W}\right)$. 
Let $(X, f)$ be any pair in $\mathcal{P}(M)$. The assignment

$$
(X, f) \mapsto \mathcal{K}(X, f)=\left(f^{-1}((-\infty, 0)), X_{\mid U}\right)
$$

defines a transformation $\mathcal{K}: \mathcal{P}(M) \rightarrow \mathcal{K}(M)$.

It is obvious from the definition of $\mathcal{K}$ that if $\left(X_{0}, f_{0}\right),\left(X_{1}, f_{1}\right) \in \mathcal{P}(M)$ are homotopic in $\mathcal{P}(M)$, then $\mathcal{K}\left(X_{0}, f_{0}\right)$ and $\mathcal{K}\left(X_{1}, f_{1}\right)$ are homotopic in $\mathcal{K}(M)$. Therefore $\mathcal{K}$ induces a transformation $\mathfrak{K}: \mathcal{P}[M] \rightarrow \mathcal{K}[M]$.

LEMMA 2.4. The mapping $\mathfrak{K}: \mathcal{P}[M] \rightarrow \mathcal{K}[M]$ is bijective.

Proof. Surjectivity. Let $(U, X) \in \mathcal{K}(M)$. Choose $\varphi$ such that $X=\nabla \varphi$. Choose an open invariant set $U_{0} \subset U$ such that $\bar{U}_{0}$ is compact and

$$
X^{-1}(0) \subset U_{0} \subset \bar{U}_{0} \subset U .
$$

Consider a smooth invariant function $f: M \rightarrow[-1,1]$ such that

$$
f(x)= \begin{cases}-1 & \text { for } x \in X^{-1}(0) \\ 1 & \text { for } x \in M \backslash U_{0}\end{cases}
$$

Choose an open invariant $U_{1} \subset U$ such that $\bar{U}_{0} \subset U_{1} \subset \bar{U}_{1} \subset U$. Consider a smooth invariant function $\mu: M \rightarrow[0,1]$ such that

$$
\mu(x)= \begin{cases}1 & \text { for } x \in \bar{U}_{0}, \\ 0 & \text { for } x \in M \backslash U_{1} .\end{cases}
$$

Set

$$
\psi(x)= \begin{cases}\mu(x) \varphi(x)+(1-\mu(x)) & \text { for } x \in X_{1}, \\ 1 & \text { for } x \in M \backslash U_{1} .\end{cases}
$$

Let $Y=\nabla \psi$ and $W=f^{-1}((-\infty, 0))$. Since $N_{-}(f) \subset U_{0}$ and $\psi(x)=\varphi(x)$ for $x \in U_{0},(Y, f) \in \mathcal{P}(M)$. By the definition of $\mathcal{K}, \mathcal{K}(Y, f)=\left(W, X_{\mid W}\right)$. According to Lemma 2.3, $(U, X)$ and $\left(W, X_{\mid W}\right)$ are homotopic in $\mathcal{K}(M)$. This completes the proof of surjectivity.

The proof of injectivity is similar and is omitted.

3. Normality conditions. Throughout this section, $(\mathbf{H})$ stands for a maximal element in $\Phi(M)$. The goal of this section is to establish a bijection

$$
\mathcal{P}[M] \approx \mathcal{P}\left[M_{(\mathbf{H})}\right] \times \mathcal{P}\left[M \backslash M_{(\mathbf{H})}\right] .
$$

In what follows, the tangent bundle $T\left(M_{(\mathbf{H})}\right)$ will be considered as a subbundle of the restriction $T(M)_{\mid M_{(\mathbf{H})}}$. Since $M_{(\mathbf{H})}$ is a $G$-invariant, closed, smooth submanifold of $M, N=T\left(M_{(\mathbf{H})}\right)^{\perp}$ is a $G$-invariant subbundle of $T(M)_{\mid M_{(\mathbf{H})}}$, called the normal bundle of $M_{(\mathbf{H})}$. We denote by $\pi: N \rightarrow M_{(\mathbf{H})}$ the bundle projection.

By the tubular neighbourhood theorem (see Theorem 2.2 of [2]) there exists an open invariant $\Omega \subset M$ and a smooth $G$-equivariant diffeomorphism $\Lambda: N \rightarrow \Omega$ which maps the zero section of $N$ onto $M_{(\mathbf{H})}$. From now 
on we identify $\Omega$ with $N$. Using this convention, for $r>0$, we denote by $D_{r}(N), B_{r}(N), S_{r}(N)$ the corresponding bundles of closed discs, open discs and spheres of radius $r$, respectively. We also put $M_{\infty}=M \backslash D_{1}(N)$. Define $X_{\nu}: N \rightarrow T(N)$ by $X_{\nu}=\nabla \nu$, where $\nu(x)=\frac{1}{2}|x|^{2}$. Clearly $X_{\nu}$ is continuous and $G$-equivariant. For $x \in N$ consider the decomposition $T_{x} M=V_{x} \oplus H_{x}$, where $V_{x}=\operatorname{Ker}(D \pi(x))$ and $H_{x}=\left(V_{x}\right)^{\perp}$.

The following result is a simple consequence of the definition of $X_{\nu}$.

REMARK 3.1. If $x \in N \backslash M_{(\mathbf{H})}$ then $X_{\nu}(x) \notin H_{x}$.

Let $\left(U_{0}, \nabla \varphi_{0}\right)$ be any pair in $\mathcal{K}\left(M_{(\mathbf{H})}\right)$ and let $\left(U_{\infty}, \nabla \varphi_{\infty}\right)$ be any pair in $\mathcal{K}\left(M_{\infty}\right)$. Define $(U, X)$ by

$$
\begin{gathered}
U:=\widehat{U}_{0} \cup U_{\infty}, \quad \text { where } \widehat{U}_{0}=\pi^{-1}\left(U_{0}\right) \cap B_{1}(N), \\
X(x)= \begin{cases}\nabla \varphi_{0}(\pi(x))+X_{\nu}(x) & \text { for } x \in \widehat{U}_{0}, \\
\nabla \varphi_{\infty}(x) & \text { for } x \in U_{\infty} .\end{cases}
\end{gathered}
$$

Note that $(D \pi(x))^{*}$ maps $T_{\pi(x)}\left(M_{(\mathbf{H})}\right)$ isomorphically onto $H_{x}$. Since

$$
\nabla\left(\varphi_{0} \circ \pi\right)(x)=(D \pi(x))^{*}\left(\nabla \varphi_{0}(\pi(x))\right),
$$

Remark 3.1 implies that if $x \in \widehat{U}_{0}$ then $X(x)=0$ if and only if $x \in M_{(\mathbf{H})}$ and $\nabla \varphi_{0}(x)=0$. Thus $(U, X) \in \mathcal{K}(M)$ and the assignment

$$
\left(\left(U_{0}, \nabla \varphi_{0}\right),\left(U_{\infty}, \nabla \varphi_{\infty}\right)\right) \mapsto(U, X)
$$

defines a transformation $\mathcal{E}: \mathcal{K}\left(M_{(\mathbf{H})}\right) \times \mathcal{K}\left(M_{\infty}\right) \rightarrow \mathcal{K}(M)$. Clearly $\mathcal{E}$ preserves $\mathcal{K}\left(M_{(\mathbf{H})}\right) \times \mathcal{K}\left(M_{\infty}\right)$-homotopy classes. Therefore it induces a transformation $\mathfrak{E}: \mathcal{K}\left[M_{(\mathbf{H})}\right] \times \mathcal{K}\left[M_{\infty}\right] \rightarrow \mathcal{K}[M]$

We can now state a theorem which plays an important role in our considerations.

THEOREM 3.1. The mapping $\mathfrak{E}: \mathcal{K}\left[M_{(\mathbf{H})}\right] \times \mathcal{K}\left[M_{\infty}\right] \rightarrow \mathcal{K}[M]$ is bijective.

Before proceeding to the proof, we introduce some notation and prove three lemmas.

LEMmA 3.1. Let $\varphi \in C_{G}^{1}(M, \mathbb{R})$. If $x_{0} \in M_{(\mathbf{H})}$ then $\nabla \varphi\left(x_{0}\right) \in T_{x_{0}}\left(M_{(\mathbf{H})}\right)$.

Proof. Since $(\mathbf{H})$ is a maximal element in $\Phi(M), M_{(\mathbf{H})}$ is a closed, $G$ invariant, smooth submanifold of $M$. Let $\Lambda: N\left(M_{(\mathbf{H})}\right) \rightarrow \Lambda\left(N\left(M_{(\mathbf{H})}\right)\right)$ be an open, $G$-invariant, tubular neighbourhood of $M_{(\mathbf{H})}$ in $M$. Since $\Lambda$ is a $G$-equivariant diffeomorphism, it preserves isotropy groups and therefore $\left(N\left(M_{(\mathbf{H})}\right)\right)_{(\mathbf{H})}=M_{(\mathbf{H})}$. Notice that $G_{\left(x_{0}, v\right)} \subset G_{x_{0}}$ for any $\left(x_{0}, v\right) \in T_{x_{0}}(M)$.

Fix $x_{0} \in M_{(\mathbf{H})}$. We claim that $\left(T_{x_{0}}(M)\right)_{(\mathbf{H})} \subset T_{x_{0}}\left(M_{(\mathbf{H})}\right)$. In fact since $\left(N\left(M_{(\mathbf{H})}\right)\right)_{(\mathbf{H})}=M_{(\mathbf{H})}$, it is clear that for $\left(x_{0}, v\right) \in N_{x_{0}}\left(M_{(\mathbf{H})}\right)$ we have $G_{\left(x_{0}, v\right)}=G_{x_{0}}$ iff $v=0$. Fix $\left(x_{0}, v, w\right) \in T_{x_{0}}(M)=T_{x_{0}}\left(N\left(M_{(\mathbf{H})}\right)\right)=$ 
$N_{x_{0}}\left(M_{(\mathbf{H})}\right) \oplus T_{x_{0}}\left(M_{(\mathbf{H})}\right)$ and notice that $G_{\left(x_{0}, v, w\right)} \subset G_{x_{0}}$. Moreover, notice that if $G_{\left(x_{0}, v, w\right)}=G_{x_{0}}$ then $v=0$. Summing up, we obtain

$$
\begin{aligned}
\left(T_{x_{0}}(M)\right)_{(\mathbf{H})} & =\left(N_{x_{0}}\left(M_{(\mathbf{H})}\right) \oplus T_{x_{0}}\left(M_{(\mathbf{H})}\right)\right)_{(\mathbf{H})} \\
& =\left(T_{x_{0}}\left(M_{(\mathbf{H})}\right)\right)_{(\mathbf{H})} \subset T_{x_{0}}\left(M_{(\mathbf{H})}\right) .
\end{aligned}
$$

Fix $\varphi \in C_{G}^{1}(M, \mathbb{R})$. It is clear that $\nabla \varphi: M \rightarrow T M$ is a continuous $G$-equivariant map. Taking into account (3.3) we obtain

$$
(\nabla \varphi)_{\mid M_{(\mathbf{H})}}: M_{(\mathbf{H})} \rightarrow\left(T(M)_{\mid M_{(\mathbf{H})}}\right)_{(\mathbf{H})} \subset T\left(M_{(\mathbf{H})}\right),
$$

which completes the proof.

Consider a function $\theta \in C^{1}(\mathbb{R}, \mathbb{R})$ satisfying the following conditions:

- $\theta(\tau)=0$ for $\tau \leq 2$,

- $\theta^{\prime}(\tau)>0$ for $2<\tau<3$,

- $\theta(\tau)=1$ for $\tau \geq 3$.

Define $\Theta: M \rightarrow M$ by

$$
\Theta(x)= \begin{cases}\theta(|x|) x & \text { for } x \in N, \\ x & \text { for } x \in M \backslash N .\end{cases}
$$

REMARK 3.2. If $|x| \leq 2$ then $\Theta(x)=\pi(x)$ and $(D \Theta(x))^{*}$ maps $T_{x} M_{(\mathbf{H})}$ isomorphically onto $H_{x}$. If $|x|>2$ then $D \Theta(x): T_{x} M \rightarrow T_{\Theta(x)} M$ is an isomorphism.

Now, consider $(U, X) \in \mathcal{K}(M)$. Choose $\varphi \in C_{G}^{1}(U, \mathbb{R})$ such that $X=\nabla \varphi$. Define $\Theta^{*}(U, X)=\left(\Theta^{-1}(U), \nabla(\varphi \circ \Theta)_{\mid \Theta^{-1}(U)}\right)$.

Lemma 3.2. For any $(U, X) \in \mathcal{K}(M)$ we have

(a) $\Theta^{*}(U, X) \in \mathcal{K}(M)$,

(b) $\Theta^{*}(U, X)$ and $(U, X)$ are $\mathcal{K}(M)$-homotopic.

Proof. (a) Let $Y=\nabla(\varphi \circ \Theta)$. We claim that for $x \in \Theta^{-1}(U)$,

$$
Y(x)=0 \Leftrightarrow X(\Theta(x))=0 .
$$

To prove this we consider the following two cases.

1. $x \in D_{2}(N) \cap \Theta^{-1}(U)$. According to the definition of $\Theta$ we have $D \Theta(x)=D \pi(x)$. Thus $D \Theta(x)$ maps $H_{x}$ isomorphically onto $T_{\Theta(x)}\left(M_{(\mathbf{H})}\right)$, and $D \Theta^{*}(x)$ maps $T_{\Theta(x)}\left(M_{(\mathbf{H})}\right)$ isomorphically onto $H_{x}$. Since

$$
\nabla(\varphi \circ \Theta)(x)=(D \Theta(x))^{*}(\nabla \varphi(\Theta(x)))
$$

and, according to Lemma 3.1, $\nabla \varphi(\Theta(x)) \in T_{\Theta(x)}\left(M_{(\mathbf{H})}\right)$, the proof of this case is complete.

2. $x \in \Theta^{-1}(U) \backslash D_{2}(N)$. In this case $D \Theta(x)$ is an isomorphism, which implies (3.4). 
Thus $Y^{-1}(0)=\Theta^{-1}\left(X^{-1}(0)\right)$. Clearly $Y^{-1}(0)$ is a compact subset of $\Theta^{-1}(U)$ and the proof of (a) is complete.

(b) For $t \in[0,1]$ define $\Theta_{t}: M \rightarrow M$ by

$$
\Theta_{t}(x)= \begin{cases}{[t \theta(|x|)+1-t] x} & \text { for } x \in N, \\ x & \text { for } x \in M \backslash N .\end{cases}
$$

Set $V=\left\{(x, t) \in M \times[0,1]: x \in \Theta_{t}^{-1}(U)\right\}$ and $Z(x, t)=\nabla\left(\varphi \circ \Theta_{t}\right)(x)$. Evidently $(V, Z)$ is a $\mathcal{K}(M)$-homotopy connecting $(U, X)$ and $\Theta^{*}(U, X)$.

Consider a function $\mu \in C^{1}(\mathbb{R}, \mathbb{R})$ satisfying the following conditions:

- $\mu(\tau)=0$ for $\tau \leq 0$,

- $\mu^{\prime}(\tau)>0$ for $0<\tau<2$,

- $\mu(\tau)=1$ for $\tau \geq 2$.

Define $\widehat{\mu} \in C_{G}^{1}(M, \mathbb{R})$ by

$$
\widehat{\mu}(x)= \begin{cases}\mu\left(\frac{1}{2}|x|^{2}\right) & \text { for } x \in N, \\ 1 & \text { for } x \in M \backslash N .\end{cases}
$$

Define $X_{\mu}: M \rightarrow T(M)$ by $X_{\mu}=\nabla \widehat{\mu}$. Clearly $X_{\mu}$ is continuous and $G$ equivariant.

Let $(U, X) \in \mathcal{K}(M)$. Define

$$
\mathcal{N}(U, X)=\left(\Theta^{-1}(U), \nabla(\varphi \circ \Theta)_{\mid \Theta^{-1}(U)}+X_{\mu \mid \Theta^{-1}(U)}\right) .
$$

Lemma 3.3. For any $(U, X) \in \mathcal{K}(M)$ we have

(a) $\mathcal{N}(U, X) \in \mathcal{K}(M)$,

(b) $\mathcal{N}(U, X)$ and $(U, X)$ are $\mathcal{K}(M)$-homotopic.

Proof. (a) Let $Y=\nabla(\varphi \circ \Theta)$. We claim that for $x \in \Theta^{-1}(U)$,

$$
\left(Y+X_{\nu}\right)^{-1}(0)=Y^{-1}(0) \cap X_{\nu}^{-1}(0) .
$$

To prove this we consider the following two cases.

- $x \in D_{2}(N) \cap \Theta^{-1}(U)$. By (3.5), $Y(x) \in H_{x}$. Applying Remark 3.1 we obtain (3.6).

- $x \in \Theta^{-1}(U) \backslash D_{2}(N)$. In this case $X_{\nu}(x)=0$, which implies (3.6).

Thus $\left(Y+X_{\nu}\right)^{-1}(0) \subset Y^{-1}(0)$, hence $\left(Y+X_{\nu}\right)^{-1}(0)$ is compact and the proof of (a) is complete.

(b) For $(x, t) \in \Theta^{-1}(U) \times[0,1]$ set $Z(x, t)=Y(x)+(1-t) X_{\nu}(x)$. Then $\left(\Theta^{-1}(U) \times[0,1], Z\right)$ is a $\mathcal{K}(M)$-homotopy connecting $\mathcal{N}(U, X)$ and $\Theta^{*}(U, X)$. By Lemma 3.2(b), $\Theta^{*}(U, X)$ and $(U, X)$ are $\mathcal{K}(M)$-homotopic and the proof is complete.

Proof of Theorem 3.1. Let $(U, \nabla \varphi) \in \mathcal{K}(M)$. Set $U_{0}=U \cap M_{(\mathbf{H})}, \varphi_{0}=$ $\varphi_{\mid U_{0}}, U_{\infty}=\Theta^{-1}(U) \cap M_{\infty}, \mathcal{R}_{0}(U, X)=\left(U_{0}, \nabla \varphi_{0}\right), \mathcal{R}_{\infty}(U, X)=\mathcal{N}(U, X)_{\mid U_{\infty}}$. 
According to Lemma 3.1, $\mathcal{R}_{0}(U, X) \in \mathcal{K}\left(M_{(\mathbf{H})}\right)$ and by the definition of $\mathcal{N}(U, \nabla \varphi), \mathcal{R}_{\infty}(U, \nabla \varphi) \in \mathcal{K}\left(M_{\infty}\right)$.

Define a map $\mathcal{R}: \mathcal{K}(M) \rightarrow \mathcal{K}\left(M_{(\mathbf{H})}\right) \times \mathcal{K}\left(M_{\infty}\right)$ by

$$
\mathcal{R}(U, X)=\left(\mathcal{R}_{0}(U, X), \mathcal{R}_{\infty}(U, X)\right) .
$$

It follows directly from the definition of $\mathcal{E}$ that $\mathcal{E}(\mathcal{R}(U, X))$ and $\mathcal{N}(U, X)$ are $\mathcal{K}(M)$-homotopic. Note that $\mathcal{R}$ preserves $\mathcal{K}(M)$-homotopy classes. Thus $\mathcal{R}$ induces a map $\mathfrak{R}: \mathcal{K}[M] \rightarrow \mathcal{K}\left[M_{(\mathbf{H})}\right] \times \mathcal{K}\left[M_{\infty}\right]$. Hence Lemma 3.3 implies that $\mathfrak{E} \circ \mathfrak{R}$ is the identity map of $\mathcal{K}[M]$.

It remains to show that $\mathfrak{R} \circ \mathfrak{E}$ equals the identity.

Let $\left(U_{0}, \nabla \varphi_{0}\right) \in \mathcal{K}\left(M_{(\mathbf{H})}\right)$ and $\left(U_{\infty}, \nabla \varphi_{\infty}\right) \in \mathcal{K}\left(M_{\infty}\right)$. Moreover, define $(U, X)=\mathcal{R}_{\infty}\left(\mathcal{E}\left(\left(U_{0}, \nabla \varphi_{0}\right),\left(U_{\infty}, \nabla \varphi_{\infty}\right)\right)\right)$. Set $U_{1}=\Theta^{-1}\left(U_{\infty}\right), X_{1}=X_{\mid U_{1}}$, $U_{2}=\Theta^{-1}\left(\pi^{-1}\left(U_{0}\right) \cap B_{1}(N)\right) \cap M_{\infty}$ and $X_{2}=X_{\mid U_{2}}$. Observe that

(a) $U=U_{1} \cup U_{2}$ and $U_{1} \cap U_{2}=\emptyset$,

(b) $\left(U_{1}, X_{1}\right),\left(U_{2}, X_{2}\right) \in \mathcal{K}\left(M_{\infty}\right)$,

(c) $X_{2}(x)=\nabla\left(\varphi_{0} \circ \pi\right)(x)+\alpha(x) X_{\nu}(x)$ for $x \in U_{2}$, where $\alpha(x) \geq 0$.

Setting $W=U_{2} \times[0,1]$ and $Y_{t}(x)=(1-t) X_{2}(x)+t X_{\nu}(x)$ for $(x, t) \in W$ we obtain a $\mathcal{K}\left(M_{\infty}\right)$-homotopy $(W, Y)$ connecting $\left(U_{2}, X_{2}\right)$ and $\left(U_{2}, Y_{1}\right)$. Clearly $Y_{1}(x) \neq 0$ for all $x \in U_{2}$. Thus, in view of (a), Lemma 2.3 implies that $(U, X)$ is homotopic to $\left(U_{1}, X_{1}\right)$ in $\mathcal{K}\left(M_{\infty}\right)$. Since $\left(U_{1}, X_{1}\right)=\mathcal{N}\left(U_{\infty}, \nabla \varphi_{\infty}\right)$, by a simple repetition of the arguments given in the proof of Lemma 3.3, we see that $\left(U_{1}, X_{1}\right)$ is homotopic to $\left(U_{\infty}, \nabla \varphi_{\infty}\right)$ in $\mathcal{K}\left(M_{\infty}\right)$. Thus we have shown that $\mathcal{R}_{\infty} \circ \mathcal{E}\left(\left(U_{0}, \nabla \varphi_{0}\right),\left(U_{\infty}, \nabla \varphi_{\infty}\right)\right)$ is homotopic to $\left(U_{\infty}, \nabla \varphi_{\infty}\right)$. Since $\mathcal{R}_{0} \circ \mathcal{E}\left(\left(U_{0}, \nabla \varphi_{0}\right),\left(U_{\infty}, \nabla \varphi_{\infty}\right)\right)=\left(U_{0}, \nabla \varphi_{0}\right), \mathcal{R} \circ \mathcal{E}$ induces the identity on $\mathcal{K}\left[M_{(\mathbf{H})}\right] \times \mathcal{K}\left[M_{\infty}\right]$. Thus the proof of Theorem 3.1 is complete.

We finish this section with a corollary which allows us to reduce the classification of $\mathcal{P}[M]$ to the classification of $\mathcal{P}\left[M_{(H)}\right]$ and $\mathcal{P}\left[M \backslash M_{(H)}\right]$, where $(H) \in \Phi(M)$. The corollary is a direct consequence of Lemmas 2.2, 2.4 and Theorem 3.1.

Corollary 3.1. Assume additionally that $\# \Phi(M)<\infty$, i.e. $\Phi(M)=$ $\left\{\left(H_{1}\right), \ldots,\left(H_{k}\right)\right\}$. Then there is a bijection

$$
\Gamma_{M}: \mathcal{P}[M] \rightarrow \mathcal{P}\left[M_{\left(H_{1}\right)}\right] \times \cdots \times \mathcal{P}\left[M_{\left(H_{k}\right)}\right] .
$$

It is worth pointing out that if the manifold $M$ is compact, then $\# \Phi(M)$ $<\infty$ (see [2], [5]).

4. Main results. In this section we prove the main result of this article. Throughout this section we assume additionally that $\# \Phi(M)<\infty$. Namely, we classify the homotopy classes $\mathcal{P}[M]$ (see Theorem 4.1). Moreover, as a corollary we obtain a classification of $\mathcal{G}[V]$. 
We start with the definition of a group $U(G, M)$. The degree we are going to construct will be an element of $U(G, M)$. Fix $(H) \in \Phi(M)$. Since $M_{(H)}$ is a smooth Riemannian $G$-manifold, $M_{(H)} / G$ is a smooth Riemannian manifold and the projection $\pi_{(H)}: M_{(H)} \rightarrow M_{(H)} / G$ is a smooth $G$-invariant map. Define $I((H), M)$

$$
\begin{gathered}
=\left\{W: W \text { is a connected component of } M_{(H)} / G \text { with } \operatorname{dim} W \geq 1\right\}, \\
U_{(H)}(G, M)=\bigoplus_{W \in I((H), M)} \mathbb{Z}, \quad U(G, M)=\bigoplus_{(H) \in \Phi(M)} U_{(H)}(G, M) .
\end{gathered}
$$

It is understood that if $I((H), M)=\emptyset$, then $U_{(H)}(G, M)=\{0\}$.

Fix $[(X, f)] \in \mathcal{P}[M]$. We will define an element

$$
\operatorname{DEG}([(X, f)], M)=\sum_{(H) \in \Phi(M)} \operatorname{DEG}_{(H)}([(X, f)], M) \in U(G, M),
$$

where $\operatorname{DEG}_{(H)}([(X, f)], M) \in U_{(H)}(G, M)$.

Suppose first that $\# \Phi(M)=1$, i.e. $\Phi(M)=\{(H)\}$. In this situation

- $M=M_{(H)}$,

- $U(G, M)=U_{(H)}\left(G, M_{(H)}\right)$,

- $\operatorname{DEG}([(X, f)], M)=\operatorname{DEG}_{(H)}\left([(X, f)], M_{(H)}\right) \in U_{(H)}\left(G, M_{(H)}\right)$.

Fix a pair $(X, f)=(\nabla \varphi, f) \in \mathcal{P}(M)$. Since $\Phi(M)=\{(H)\}, M / G$ is a smooth manifold and the quotient map $\pi_{(H)}: M \rightarrow M / G$ is smooth. Define functions $\varphi_{0}, f_{0} \in C^{2}(M / G, \mathbb{R})$ such that $\varphi(x)=\left(\varphi_{0} \circ \pi_{(H)}\right)(x)$ and $f(x)=$ $\left(f_{0} \circ \pi_{(H)}\right)(x)$.

Notice that if $W \in I((H), M)$, then the group $G$ acts trivially on $W$.

It is clear that if $W \in I((H), M)$, then $\left[\left(\nabla \varphi_{0}, f_{0}\right)_{\mid W}\right] \in \mathcal{P}[W]$. Moreover, it is easy to check that the map

$$
P_{(H)}: \mathcal{P}[M] \rightarrow \mathcal{P}[M / G]=\prod_{W \in I((H), M)} \mathcal{P}[W]
$$

defined by $P_{(H)}([(X, f)])=\prod_{W \in I((H), M)}\left[\left(\nabla \varphi_{0}, f_{0}\right)_{\mid W}\right]$ is a bijection. On the other hand, we have a bijection

$$
\gamma_{(H)}=\sum_{W \in I((H), M)} \gamma_{W}: \prod_{W \in I((H), M)} \mathcal{P}[W] \rightarrow U(G, M)=\bigoplus_{W \in I((H), M)} \mathbb{Z},
$$

where the bijections $\gamma_{W}: \mathcal{P}[W] \rightarrow \mathbb{Z}$ are given by Corollary 6.2. Finally, we define a bijection $\operatorname{DEG}_{(H)}(\cdot, M): \mathcal{P}(M) \rightarrow U(G, M)$ by

$$
\operatorname{DEG}_{(H)}([(X, f)], M):=\left(\gamma_{(H)} \circ P_{(H)}\right)([(X, f)]) .
$$

Suppose now that $\# \Phi(M)>1$, i.e. $\Phi(M)=\left\{\left(H_{1}\right), \ldots,\left(H_{k}\right)\right\}, k>1$. In this situation we define a bijection $\operatorname{DEG}(\cdot, M): \mathcal{P}[M] \rightarrow U(G, M)$ as 
follows:

$\operatorname{DEG}([(X, f)], M)$

$$
=\left(\left(\mathrm{DEG}_{\left(H_{1}\right)}\left(\cdot, M_{\left(H_{1}\right)}\right), \ldots, \mathrm{DEG}_{\left(H_{k}\right)}\left(\cdot, M_{\left(H_{k}\right)}\right)\right) \circ \Gamma_{M}\right)([(X, f)]),
$$

where $\Gamma_{M}: \mathcal{P}[M] \rightarrow \mathcal{P}\left[M_{\left(H_{1}\right)}\right] \times \cdots \times \mathcal{P}\left[M_{\left(H_{k}\right)}\right]$ is the bijection given by Corollary 3.1.

Notice that we have just proved the following theorem.

THEOREM 4.1. Let $G$ be any compact Lie group and let $M$ be a smooth Riemannian G-manifold such that $\# \Phi(M)<\infty$. Then the above defined map $\operatorname{DEG}(\cdot, M): \mathcal{P}[M] \rightarrow U(G, M)$ is a bijection.

Since $S(V) \subset V$ is a compact, Riemannian $G$-manifold, \# $\#(S(V))<\infty$. Therefore combining Lemma 2.1 with Theorem 4.1 we obtain the following corollary.

COROLlaRY 4.1. Let $V$ be an orthogonal representation of a compact Lie group $G$. Then the map $\nabla_{G}$-deg $: \mathcal{G}[V] \rightarrow U(G, S(V))$ defined by

$$
\nabla_{G^{-}} \operatorname{deg}([\nabla F], B(V))=\operatorname{DEG}\left(\Upsilon_{V}([\nabla F], S(V))\right)
$$

is a bijection.

REMARK 4.1. It is easy to check that $\nabla_{G^{-}} \operatorname{deg}=\operatorname{DEG}(\cdot, S(V)) \circ \Upsilon_{V}$ is nothing other than the degree for $G$-equivariant gradient maps defined in [6].

5. Final remarks. Let $F_{0}, F_{1} \in C_{G}^{2}(V, \mathbb{R})$ be such that $\left(\nabla F_{i}\right)^{-1}(0) \cap$ $D(V)=\{0\}$ and $\operatorname{det} \nabla^{2} F_{i}(0) \neq 0$ for $i=0,1$. Moreover, assume that

$$
\nabla_{G^{-}} \operatorname{deg}\left(\left[\nabla F_{0}\right], B(V)\right)=\nabla_{G^{-}} \operatorname{deg}\left(\left[\nabla F_{1}\right], B(V)\right) .
$$

From Corollary 4.1 it follows that there exists a family of potentials $F_{t} \in$ $C_{G}^{2}(V, \mathbb{R}), t \in[0,1]$, such that the maps $\nabla F_{0}, \nabla F_{1}$ are joined by a $\mathcal{G}(V)$ homotopy $\nabla_{v} F_{t}:(D(V), S(V)) \rightarrow(V, V \backslash\{0\}), t \in[0,1]$. Let $\phi \in C_{G}^{2}(V, \mathbb{R})$ be such that there are $0<\alpha, \varepsilon<1$ such that

- $D_{\alpha}(V) \subset \phi^{-1}(1)$,

- $\operatorname{cl}\left(D_{1+\varepsilon}(V) \backslash D_{1-\varepsilon}(V)\right) \subset \phi^{-1}(0)$.

Finally, define a family of potentials $H_{t} \in C_{G}^{2}(V, \mathbb{R}), t \in[0,1]$, as follows:

$$
H_{t}(v)=(1-\phi(v)) \cdot F_{t}(v)+\frac{1}{2} \cdot \phi(v) \cdot\left\langle\nabla^{2} F_{t}(0) v, v\right\rangle .
$$

Notice that $\nabla_{v} H_{t}: V \rightarrow V, t \in[0,1]$, is a $\mathcal{G}(V)$-homotopy such that

- $\nabla_{v} H_{t}(0)=0$ for any $t \in[0,1]$,

- $\left(\nabla_{v} H_{i}(0)\right)^{-1}(0) \cap D(V)=\{0\}$ for $i=0,1$,

- $\nabla_{v}^{2} H_{t}(0)$ is a continuous family of self-adjoint $G$-equivariant linear maps joining $\nabla_{v}^{2} F_{0}(0)$ and $\nabla_{v}^{2} F_{1}(0)$. 
Thus there is no global bifurcation of connected sets of non-trivial solutions of the equation $\nabla_{v} H_{t}(v)=0$ off $\{0\} \times[0,1]$. However, there is local bifurcation off $\{0\} \times[0,1]$ if $i_{G}\left(\nabla_{v}^{2} F_{0}(0), 0\right) \neq i_{G}\left(\nabla_{v}^{2} F_{1}(0), 0\right)$, i.e. the $G$-equivariant Conley indices of the origin are different. This can be proved by the Conley index techniques. Thus the degree for $G$-equivariant gradient maps is the natural invariant to study global bifurcation of zeros for $G$-equivariant gradient mappings.

It would be of interest to know if there is an example where there are no non-trivial connected sets of non-trivial solutions intersecting $\{0\} \times[0,1]$ as in the work of Böhme [1].

We need to be careful in the interpretation of this phenomenon. For gradient maps, it is well known that the Conley index provides stronger invariants for homotopy classes of gradient maps. But this only applies when not only the critical points but also the connecting orbits joining them do not cross the boundary of the neighbourhood we are working in. We can actually do a little better, but this does not matter for our present discussion. In particular, suppose that $\nabla F_{0}, \nabla F_{1}: V \rightarrow V$ are gradient $G$-equivariant mappings such that $\nabla F_{0}(v) \neq 0$ and $\nabla F_{1}(v) \neq 0$ if $\|v\| \geq 1$.

If $\nabla_{G^{-}} \operatorname{deg}\left(\left[\nabla F_{0}\right], B(V)\right)=\nabla_{G^{-}} \operatorname{deg}\left(\left[\nabla F_{1}\right], B(V)\right)$ then, by Corollary 4.1, $\left[\nabla F_{0}\right]=\left[\nabla F_{1}\right]$, i.e. $\nabla F_{0}, \nabla F_{1}$ are homotopic through a $G$-equivariant gradient homotopy $\nabla F_{t}, t \in[0,1]$, such that $\nabla F_{t}(v) \neq 0$ if $\|v\|=1$.

Now suppose that $\nabla F_{0}$ has the property that all the connecting orbits of $\nabla F_{0}$ joining zeros of $\nabla F_{0}$ lie in $\|x\|<1$ and $\nabla F_{1}$ has the same property. If $\nabla F_{0}$ and $\nabla F_{1}$ have different $G$-equivariant Conley indices on the unit ball, it follows that for any equivariant homotopy $F_{t}, t \in[0,1]$, joining $\nabla F_{0}$ and $\nabla F_{1}$ we have no uniform bound on the zeros of $F_{t}$ and connecting orbits (otherwise the Conley indices on large balls would be the same). Hence we see that the homotopy theory of gradient maps is quite different when we bound only the zeros, or both the zeros and the connecting orbits.

6. Appendix. In this section we classify the homotopy classes of gradient pairs on a connected smooth Riemannian manifold $N$. Unless otherwise stated, throughout this section we assume that $\operatorname{dim} N>1$. The proof is similar in spirit to that of [8].

In the first step we reduce the classification of homotopy classes of gradient pairs on a manifold $N$ to the classification of admissible gradient maps on $\mathbb{R}^{\operatorname{dim} N}$ (see Theorem 6.1).

In the second step, for the convenience of the reader, we briefly sketch the classification of admissible gradient maps on $\mathbb{R}^{\operatorname{dim} N}$, due to Parusiński [8] (see Theorem 6.4).

Finally, in Corollary 6.2 we classify the homotopy classes of gradient pairs on a connected smooth Riemannian manifold $N$. 
We keep the notation of the previous sections, although in this section $G=\{e\}$.

Let $B_{\alpha}^{n}=\left\{x \in \mathbb{R}^{n}:|x|<\alpha\right\}, D_{\alpha}^{n}=\operatorname{cl}\left(B_{\alpha}^{n}\right)$ and $S_{\alpha}^{n-1}=\partial B_{\alpha}^{n}$. We write $B^{n}, D^{n}, S^{n-1}$ instead of $B_{1}^{n}, D_{1}^{n}, S_{1}^{n-1}$, respectively.

The principal significance of Theorem 6.1 is that it allows us to reduce the classification of $\mathcal{P}[N]$ to the classification of $\mathcal{G}\left[\mathbb{R}^{\operatorname{dim} N}\right]$.

In order to prove this theorem we first need two technical lemmas. We leave their proofs to the reader.

Lemma 6.1. Let $(\nabla \varphi, f) \in \mathcal{P}(N)$. If $(U, h)$ is a chart on $N$ such that

- $h^{-1}\left(D^{\operatorname{dim} N}\right) \subset U$,

- $\operatorname{cl}(U) \cap N_{-}(f)=\emptyset$,

- $\varphi$ is a Morse function on $h^{-1}\left(D^{\operatorname{dim} N}\right)$,

then $(\nabla \varphi, f)$ is $\mathcal{P}(N)$-homotopic to a pair $(\nabla \psi, f) \in \mathcal{P}(N)$ such that

- $(\nabla \psi)^{-1}(0) \cap h^{-1}\left(D^{\operatorname{dim} N}\right)=\emptyset$,

- $\nabla \varphi=\nabla \psi$ on $N_{-}(f)$.

Lemma 6.2. Let $(\nabla \varphi, f) \in \mathcal{P}(N)$. If $(U, h)$ is a chart on $N$ such that

- $h^{-1}\left(D^{\operatorname{dim} N}\right) \subset U$,

- $\operatorname{cl}(U) \cap N_{-}(f)=\emptyset$,

- $(\nabla \varphi)^{-1}(0) \cap h^{-1}\left(D^{\operatorname{dim} N}\right)=\emptyset$,

- $\varphi$ is a Morse function on $N_{-}(f)$,

then the pair $(\nabla \varphi, f)$ is $\mathcal{P}(N)$-homotopic to a pair $(\nabla \psi, g) \in \mathcal{P}(N)$ such that

- $N_{-}(g)=h^{-1}\left(D^{\operatorname{dim} N}\right)$,

- $\psi$ is a Morse function on $N_{-}(g)$,

- $g^{-1}(0)=h^{-1}\left(S^{\operatorname{dim} N-1}\right)$.

The following theorem is the first step in the classification of $\mathcal{P}[N]$.

Theorem 6.1. There is a bijection $j_{N}: \mathcal{P}[N] \rightarrow \mathcal{G}\left[\mathbb{R}^{\operatorname{dim} N}\right]$.

Proof. Fix $[(\nabla \varphi, f)] \in \mathcal{P}(N)$. It is easy to show that there is $\varphi_{1} \in$ $C^{2}(N, \mathbb{R})$ such that

- $\varphi_{1}$ is a Morse function on $N_{-}(f) \backslash f^{-1}(0)$,

- $\varphi_{1}(x)=\varphi(x)$ on $N \backslash\left(N_{-}(f) \backslash f^{-1}(0)\right)$.

It is evident that $\left(\nabla\left(t \cdot \varphi_{1}+(1-t) \cdot \varphi\right), f\right)$ is a well defined $\mathcal{P}(N)$-homotopy joining $(\nabla \varphi, f)$ to $\left(\nabla \varphi_{1}, f\right)$. Let $(U, h)$ be a chart on $N$ such that $\operatorname{cl}(U) \cap$ $N_{-}(f)=\emptyset$. There is no loss of generality in assuming that $h^{-1}\left(D^{\operatorname{dim} N}\right) \subset U$. It is clear that there is $\varphi_{2} \in C^{2}(N, \mathbb{R})$ such that

- $\varphi_{2}(x)=\varphi_{1}(x)$ for $x \in N \backslash U$,

- $\varphi_{2}$ is regular on $N_{-}(f) \cup h^{-1}\left(D^{\operatorname{dim} N}\right)$. 
It is easy to verify that $\left(\nabla\left(t \cdot \varphi_{2}+(1-t) \cdot \varphi_{1}\right), f\right)$ is a well defined $\mathcal{P}(N)$-homotopy joining $\left(\nabla \varphi_{1}, f\right)$ to $\left(\nabla \varphi_{2}, f\right)$. By Lemma 6.1 the gradient pair $\left(\nabla \varphi_{2}, f\right)$ is $\mathcal{P}(N)$-homotopic to a pair $\left(\nabla \varphi_{3}, f\right)$ such that

- $X_{3}^{-1}(0) \cap h^{-1}\left(D^{\operatorname{dim} N}\right)=\emptyset$,

- $\varphi_{3}$ is a Morse function on $N_{-}(f)$.

From Lemma 6.2 it follows that there is a gradient pair $\left(\nabla \varphi_{4}, f_{4}\right) \mathcal{P}(N)$-homotopic to the pair $\left(\nabla \varphi_{3}, f\right)$ such that

- $N_{-}\left(f_{4}\right)=h^{-1}\left(D^{\operatorname{dim} N}\right)$,

- $f_{4}^{-1}(0)=h^{-1}\left(S^{\operatorname{dim} N-1}\right)$,

- $\varphi_{4}$ is a Morse function on $N_{-}\left(f_{4}\right)$.

Let $\phi: \mathbb{R}^{\operatorname{dim} N} \rightarrow \mathbb{R}$ be any $C^{1}$-extension of $\varphi_{4} \circ h^{-1}: D^{\operatorname{dim} N} \rightarrow \mathbb{R}$. We define a map $j_{N}: \mathcal{P}[N] \rightarrow \mathcal{G}\left[\mathbb{R}^{\operatorname{dim} N}\right]$ by $j_{N}([(\nabla \varphi, f)])=[\nabla \phi]$. Directly from the definition it follows that $j_{N}$ is well defined. What is left is to show that $j_{N}$ is a bijection.

Define a map $k_{N}: \mathcal{G}\left[\mathbb{R}^{\operatorname{dim} N}\right] \rightarrow \mathcal{P}[N]$ in the following way. Let $(U, h)$ be a chart on $N$ such that $h^{-1}\left(D_{1+\varepsilon}^{\operatorname{dim} N}\right) \subset U$. Fix $\nabla \varphi \in \mathcal{G}\left(\mathbb{R}^{\operatorname{dim} N}\right)$ and denote by $\psi_{\nabla \varphi} \in C^{1}(N, \mathbb{R})$ any extension of $\varphi \circ h: h^{-1}\left(D_{1+\varepsilon}^{\operatorname{dim} N}\right) \rightarrow \mathbb{R}$. Finally, choose any $f_{\nabla \varphi} \in C^{0}(N, \mathbb{R})$ such that $N_{-}\left(f_{\nabla \varphi}\right)=h^{-1}\left(D^{\operatorname{dim} N}\right)$ and $f_{\nabla \varphi}^{-1}(0)=$ $h^{-1}\left(S^{\operatorname{dim} N-1}\right)$. Define $k_{N}: \mathcal{G}\left[\mathbb{R}^{\operatorname{dim} N}\right] \rightarrow \mathcal{P}[N]$ by $k_{N}[\nabla \varphi]=\left[\left(\nabla \psi_{\nabla \varphi}, f_{\nabla \varphi}\right)\right]$. It is clear that $k_{N}$ is well defined. We check at once that $k_{N}$ is the inverse of $j_{N}$, which completes the proof.

Let $\varphi \in C^{2}(N, \mathbb{R})$ be a Morse function such that $\nabla \varphi(p)=0$. Then we denote by $m^{-}(p, \nabla \varphi) \in \mathbb{N} \cup\{0\}$ the Morse index of $p$. If $\Omega \subset \mathbb{R}^{n}$ is an open and bounded subset, and moreover $q \in C^{0}\left(\operatorname{cl}(\Omega), \mathbb{R}^{n}\right)$ is such that $q^{-1}(0) \cap \partial \Omega=\emptyset$ then $\operatorname{deg}(q, \Omega, 0) \in \mathbb{Z}$ denotes the Brouwer degree.

Lemma 6.3. Let $(\nabla \varphi, f) \in \mathcal{P}(N)$ be such that $\varphi \in C^{2}(N, \mathbb{R})$ is a Morse function on $N_{-}(f)$. Then

$$
\operatorname{deg}\left(j_{N}([(\nabla \varphi, f)]), B^{n}, 0\right)=\sum_{p \in N_{-}(f) \cap(\nabla \varphi)^{-1}(0)}(-1)^{m^{-}(p, \nabla \varphi)} .
$$

Proof. The easy proof is left to the reader.

From Theorem 6.1 it follows that to classify $\mathcal{P}[N]$ it is enough to classify $\mathcal{G}\left[\mathbb{R}^{\operatorname{dim} N}\right]$. In fact $\mathcal{G}\left[\mathbb{R}^{\operatorname{dim} N}\right]$ has been classified in [8]. Below we recall the main steps of this proof.

Let $\nabla F \in \mathcal{G}\left(\mathbb{R}^{n}\right)$. For $x \in S^{n-1}$ we have the unique decomposition

$$
\begin{aligned}
\nabla F(x) & =(\nabla F(x)-\langle\nabla F(x), x\rangle \cdot x)+\langle\nabla F(x), x\rangle \cdot x \\
& =X_{\nabla F}(x)+f_{\nabla F}(x) \cdot x .
\end{aligned}
$$

It is clear that $\left(X_{\nabla F}, f_{\nabla F}\right)=\left[\left(\nabla\left(F_{\mid S^{n-1}}\right), f_{\nabla F}\right)\right] \in \mathcal{P}\left(S^{n-1}\right)$. 
Lemma 6.4. The map $i_{n}: \mathcal{G}\left[\mathbb{R}^{n}\right] \rightarrow \mathcal{P}\left[S^{n-1}\right]$ defined by $i_{n}([\nabla F])=$ $\left[\left(X_{\nabla F}, f_{\nabla F}\right)\right]$ is a bijection.

LEMma 6.5. If $F_{\mid S^{n-1}} \in C^{2}\left(S^{n-1}, \mathbb{R}\right)$ is a Morse function, then

$$
\operatorname{deg}\left([\nabla F], B^{n}, 0\right)=1-\sum_{p \in N_{-}\left(f_{\nabla F}\right) \cap X_{\nabla F}^{-1}(0)}(-1)^{m^{-}\left(p, X_{\nabla F}\right)} .
$$

As a direct consequence of Theorem 6.1 and Lemmas 6.4, 6.5 we obtain the following corollary.

Corollary 6.1. Fix any $n \geq 2$. Then

(1) the map $\mathcal{G}\left[\mathbb{R}^{n+1}\right] \stackrel{i_{n+1}}{\longrightarrow} \mathcal{P}\left[S^{n}\right] \stackrel{j_{S^{n}}}{\longrightarrow} \mathcal{G}\left[\mathbb{R}^{n}\right]$ is a bijection,

(2) for $\nabla F \in \mathcal{G}\left[\mathbb{R}^{n+1}\right]$,

$$
\operatorname{deg}\left(\left(j_{S^{n}} \circ i_{n+1}\right)[\nabla F], B^{n}, 0\right)=1-\operatorname{deg}\left([\nabla F], B^{n+1}, 0\right) .
$$

From Corollary 6.1 it follows that to classify $\mathcal{G}\left[\mathbb{R}^{n+1}\right]$ it is enough to classify $\mathcal{P}\left[S^{1}\right]$.

THEOREM 6.2. There is a bijection $j_{S^{1}}: \mathcal{P}\left[S^{1}\right] \rightarrow \mathbb{Z}$. Moreover, if $\left(\varphi^{\prime}, f\right) \in \mathcal{P}\left(S^{1}\right)$ and $\varphi \in C^{2}\left(S^{1}, \mathbb{R}\right)$ is a Morse function then

$$
j_{S^{1}}\left(\left[\left(\varphi^{\prime}, f\right)\right]\right)=\sum_{p \in N_{-}(f) \cap\left(\varphi^{\prime}\right)^{-1}(0)}(-1)^{m^{-}\left(p, \varphi^{\prime}\right)} .
$$

Furthermore, $j_{S^{1}}([(\sin k t, \cos k t)])=k$.

We can also classify the gradient pairs on an open interval.

TheOREM 6.3. There is a bijection $j_{(0,2 \pi)}: \mathcal{P}[(0,2 \pi)] \rightarrow \mathbb{Z}$. If $\left(\varphi^{\prime}, f\right) \in$ $\mathcal{P}((0,2 \pi))$ and $\varphi \in C^{2}((0,2 \pi), \mathbb{R})$ is a Morse function then

$$
j_{(0,2 \pi)}\left(\left[\left(\varphi^{\prime}, f\right)\right]\right)=\sum_{p \in N_{-}(f) \cap\left(\varphi^{\prime}\right)^{-1}(0)}(-1)^{m^{-}\left(p, \varphi^{\prime}\right)} .
$$

Moreover, $j_{(0,2 \pi)}([(\sin k t, \cos k t)])=k$.

The following theorem is a direct consequence of Lemma 6.3.

ThEOREM 6.4. For $k>1$ the bijection

$$
\delta_{k} \circ j_{S^{1}} \circ i_{2} \circ j_{S^{2}} \circ i_{3} \circ \ldots \circ j_{S^{k-1}} \circ i_{k}: \mathcal{G}\left[\mathbb{R}^{k}\right] \rightarrow \mathbb{Z}
$$

is given by

$$
\left(\delta_{k} \circ j_{S^{1}} \circ i_{2} \circ j_{S^{2}} \circ i_{3} \circ \ldots \circ j_{S^{2 k-1}} \circ i_{2 k}\right)([\nabla F])=\operatorname{deg}\left([\nabla F], B^{k}, 0\right),
$$

where the bijection $\delta_{k}: \mathbb{Z} \rightarrow \mathbb{Z}$ is defined by

$$
\delta_{k}(m)= \begin{cases}1-m & \text { if } k \text { is even } \\ m & \text { otherwise. }\end{cases}
$$


Finally, we classify the homotopy classes of gradient pairs on a connected smooth Riemannian manifold $N$.

COROLlaRY 6.2. Let $N$ be a connected smooth Riemannian manifold with $\operatorname{dim} N \geq 1$. Then there is a bijection $\Upsilon_{N}: \mathcal{P}[N] \rightarrow \mathbb{Z}$ given by

$$
\Upsilon_{N}([(\nabla \varphi, f)])=\sum_{p \in N_{-}(f) \cap(\nabla \varphi)^{-1}(0)}(-1)^{m^{-}(p, \nabla \varphi)},
$$

where $\varphi \in C^{2}(N, \mathbb{R})$ is a Morse function.

Proof. We define $\Upsilon_{N}: \mathcal{P}[N] \rightarrow \mathbb{Z}$ as follows:

$$
\Upsilon_{N}= \begin{cases}\delta_{\operatorname{dim} N} \circ j_{S^{1}} \circ i_{2} \circ j_{S^{2}} \circ i_{3} \circ \ldots \circ j_{S^{\operatorname{dim} N-1}} \circ i_{\operatorname{dim} N} \circ j_{N} \\ j_{S^{1}} & \text { if } \operatorname{dim} N>1, \\ j_{(0,2 \pi)} & \text { if } N \approx S^{1}, \\ & \text { if } N \approx(0,2 \pi) .\end{cases}
$$

Fix $[(\nabla \varphi, f)] \in \mathcal{P}(N)$. Without loss of generality one can assume that $\varphi \in$ $C^{2}(N, \mathbb{R})$ is a Morse function. The rest of the proof is a consequence of Lemma 6.3 and Theorems 6.2-6.4.

\section{References}

[1] R. Böhme, Die Lösung der Verzweigungsgleichungen für nichtlineare Eigenwertprobleme, Math. Z. 127 (1972), 105-126.

[2] G. E. Bredon, Introduction to Compact Transformation Groups, Academic Press, New York, 1972.

[3] E. N. Dancer, A new degree for $S^{1}$-invariant gradient mappings and applications, Ann. Inst. H. Poincaré Anal. Non Linéaire 2 (1985), 329-370.

[4] T. tom Dieck, Transformation Groups, de Gruyter, Berlin, 1987.

[5] J. J. Duistermaat and J. A. C. Kolk, Lie Groups, Springer, Berlin, 2000.

[6] K. Gęba, Degree for gradient equivariant maps and equivariant Conley index, in: Topological Nonlinear Analysis, Degree, Singularity and Variations, M. Matzeu and A. Vignoli (eds.), Progr. Nonlinear Differential Equations Appl. 27, Birkhäuser, 1997, 247-272.

[7] K. Gęba, I. Massabó and A. Vignoli, On the Euler characteristic of equivariant vector fields, Boll. Un. Mat. Ital. 4 (1990), 243-251.

[8] A. Parusiński, Gradient homotopies of gradient vector fields, Studia Math. 96 (1990), 73-80.

[9] R. Rubinsztein, On the equivariant homotopy of spheres, Dissertationes Math. 134 (1976).

[10] S. Rybicki, $A$ degree for $S^{1}$-equivariant orthogonal maps and its applications to bifurcation theory, Nonlinear Anal. 23 (1994), 83-102.

[11] -, On Rabinowitz alternative for the Laplace-Beltrami operator on $S^{n-1}$. Continua that meet infinity, Differential Integral Equations 9 (1996), 1267-1277. 
[12] S. Rybicki, Global bifurcations of solutions of Emden-Fowler type equation $-\Delta u(x)$ $=\lambda f(u(x))$ on an annulus in $\mathbb{R}^{n}, n \geq 3$, J. Differential Equations 183 (2002), $208-223$.

School of Mathematics

Sydney University

Sydney, NSW 2006

Australia

E-mail: normd@math.usyd.edu.au

Faculty of Technical Physics

and Applied Mathematics

Technical University of Gdańsk

Narutowicza 11-12

Faculty of Mathematics

PL-80-952 Gdańsk, Poland

E-mail: geba@mifgate.mif.pg.gda.pl

and Computer Science

Nicolaus Copernicus University

Chopina 12-18

PL-87-100 Toruń, Poland

E-mail: Slawomir.Rybicki@mat.uni.torun.pl

Received 26 May 2003;

in revised form 27 April 2004 and 16 February 2005 